\title{
Complementary Cycles in Irregular Multipartite Tournaments
}

\author{
Zhihong He, ${ }^{1}$ Xiaoying Wang, ${ }^{2}$ and Caiming Zhang ${ }^{3}$ \\ ${ }^{1}$ School of Mathematics and Information Science, Yantai University, Yantai 264005, China \\ ${ }^{2}$ Chifeng University, Chifeng 024000, China \\ ${ }^{3}$ College of Computer Science and Technology, Shandong University, Jinan 250101, China
}

Correspondence should be addressed to Zhihong He; zhihhe@126.com

Received 29 March 2016; Accepted 17 July 2016

Academic Editor: Sergii V. Kavun

Copyright (C) 2016 Zhihong He et al. This is an open access article distributed under the Creative Commons Attribution License, which permits unrestricted use, distribution, and reproduction in any medium, provided the original work is properly cited.

\begin{abstract}
A tournament is a directed graph obtained by assigning a direction for each edge in an undirected complete graph. A digraph $D$ is cycle complementary if there exist two vertex disjoint cycles $C$ and $C^{\prime}$ such that $V(D)=V(C) \cup V\left(C^{\prime}\right)$. Let $D$ be a locally almost regular $c$-partite tournament with $c \geq 3$ and $|\gamma(D)| \leq 3$ such that all partite sets have the same cardinality $r$, and let $C_{3}$ be a 3 -cycle of $D$. In this paper, we prove that if $D-V\left(C_{3}\right)$ has no cycle factor, then $D$ contains a pair of disjoint cycles of length 3 and $|V(D)|-3$, unless $D$ is isomorphic to $T_{7}, D_{4,2}, D_{4,2}^{*}$, or $D_{3,2}$.
\end{abstract}

\section{Introduction}

Denote the vertex set and arc set of a digraph $D$ by $V(D)$ and $E(D)$, respectively. For a subset $X$ of $V(D)$, we define $D[X]$ as the subdigraph induced by $X$. If $x y$ is an arc of a digraph $D$, then we write $x \rightarrow y$ and say $x$ dominates $y$. If $X$ and $Y$ are two disjoint subsets of $V(D)$ or subdigraphs of $D$ such that every vertex of $X$ dominates every vertex of $Y$, then $X$ dominates $Y$, denoted by $X \rightarrow Y$. We write $X \Rightarrow Y$ if there is no arc from $Y$ to $X$.

The out-neighborhood $N_{D}^{+}(x)=N^{+}(x)$ of a vertex $x$ is the set of vertices dominated by $x$, and the in-neighborhood $N_{D}^{-}(x)=N^{-}(x)$ is the set of vertices dominating $x$. The numbers $d_{D}^{+}(x)=d^{+}(x)=\left|N^{+}(x)\right|$ and $d_{D}^{-}(x)=d^{-}(x)=$ $\left|N^{-}(x)\right|$ are the outdegree and indegree of $x$, respectively. The minimum outdegree and the minimum indegree of $D$ are denoted by $\delta^{+}(D)=\delta^{+}$and $\delta^{-}(D)=\delta^{-}$and $\delta(D)=$ $\delta=\min \left\{\delta^{+}, \delta^{-}\right\}$. The local irregularity is defined by $i_{l}(D)=$ $\max \left|d^{+}(x)-d^{-}(x)\right|$ over all vertices $x$ of $D$ and the global irregularity is defined by $i_{g}(D)=\max \left\{\max \left(d^{+}(x), d^{-}(x)\right)-\right.$ $\left.\min \left(d^{+}(y), d^{-}(y)\right) \mid x, y \in V(D)\right\}$. Clearly, $i_{l}(D) \leq i_{g}(D)$. If $i_{g}(D)=0$, then $D$ is regular and if $i_{g}(D) \leq 1$, then $D$ is almost regular. If $i_{l}(D) \leq 1$, then $D$ is locally almost regular.

By a path or cycle we mean a directed path or cycle. A cycle of length $m$ is an $m$-cycle. The independence number $\alpha(D)=\alpha$ is the maximum size among all independent sets of vertices of $D$. A digraph $D$ is strongly connected or strong if, for each pair of vertices $u$ and $v$, there is a path from $u$ to $v$ in $D$. A digraph $D$ with at least $k+1$ vertices is $k$-connected if for any set $A$ of at most $k-1$ vertices, the subdigraph $D-A$ obtained by deleting $A$ is strong. The connectivity of $D$, denoted by $\kappa(D)$, is defined to be the largest value of $k$ such that $D$ is $k$ connected.

A cycle factor is a spanning subdigraph consisting of disjoint cycles. A cycle factor with the minimum number of cycles is called a minimal cycle factor. If we replace every arc $x y$ of $D$ by $y x$, then we call the resulting digraph, denoted by $D^{-1}$, the converse digraph of $D$.

A $c$-partite graph is a graph whose vertices are or can be partitioned into $c$ different independent sets. Equivalently, it is a graph that can be colored with $c$ colors, so that no two endpoints of an edge have the same color. A complete $c$-partite graph is a graph that can be partitioned into $c$ independent sets, as above, so that every pair of vertices from two different independent sets have different colors.

A c-partite or multipartite tournament is an orientation of a complete $c$-partite graph. If $D$ is a $c$-partite tournament with the partite sets $V_{1}, V_{2}, \ldots, V_{c}$ such that $\left|V_{1}\right| \leq\left|V_{2}\right| \leq \cdots \leq\left|V_{c}\right|$, then we define $\gamma(D)=\left|V_{1}\right|$. If the vertex $x$ of $D$ belongs to the partite set $V_{i}$, then we define $V(x)=V_{i}$.

A digraph $D$ is cycle complementary if there exist two vertex disjoint cycles $C$ and $C^{\prime}$ such that $V(D)=V(C) \cup V\left(C^{\prime}\right)$. 
The problem of complementary cycles in tournaments was almost completely solved by Reid [1] in 1985 and by Song [2] in 1993. The authors proved that every 2-connected tournament $D$ on at least 8 vertices has complementary cycles of length $t$ and $|V(D)|-t$ for all $t \in\{3,4, \ldots,|V(D)|-3\}$.

There exists only the following conjecture.

Conjecture 1 (see Yeo [3] 1999). A regular c-partite tournament $D$ with $c \geq 4$ and $|V(D)| \geq 8$ has a pair of vertex disjoint cycles of length $t$ and $|V(D)|-t$ for all $t \in\{3,4, \ldots,|V(D)|-3\}$.

In 2005, Volkmann [4] confirmed the first conjecture for $t=3$, unless $D$ is a regular 4-partite tournament with two vertices in each partite set. In 2004, Volkmann [5] confirmed conjecture for $t=4$. In 2009, He et al. [6] confirmed conjecture for $t=5$. In 2013, Volkmann [7] confirmed every almost regular $c$-partite tournament $D$ with $|V(D)| \geq 8$ such that all partite sets have the same cardinality $r$. If $c \geq 5$ or $c=4$ and $r \geq 6$, then $D$ contains a pair of vertex disjoint directed cycles of lengths 4 and $|V(D)-4|$. In 2014, Bai et al. [8] confirmed every regular bipartite tournament contains two complementary cycles. In 2014, He et al. [9] characterized all locally almost regular multipartite tournaments $D$ whose partite sets have the same cardinality with $\gamma(D) \geq 4$ that contain two complementary cycles of length 3 and $|V(D)|-3$. Let $D$ be a locally almost regular $c$-partite tournament with $c \geq 3$ and $|\gamma(D)| \leq 3$ such that all partite sets have the same cardinality, and let $C_{3}$ be a 3-cycle of $D$. We prove if $D-V\left(C_{3}\right)$ has no cycle factor, then $D$ contains a pair of disjoint cycles of length 3 and $|V(D)|-3$, unless $D$ is isomorphic to $T_{7}, D_{4,2}, D_{4,2}^{*}$, or $D_{3,2}$.

\section{Preliminary Results}

The following results play important roles in the proof of our main theorem (Theorem 12), where the first one is immediate.

Lemma 2 (see [7]). If $D$ is a regular c-partite tournament with the partite sets $V_{1}, V_{2}, \ldots, V_{c}$, then $\alpha(D)=\left|V_{1}\right|=\left|V_{2}\right|=\cdots=$ $\left|V_{c}\right|$.

Lemma 3 (see Yeo [10] 1998). If D is a multipartite tournament, then

$$
\kappa(D) \geq\left\lceil\frac{|V(D)|-2 i_{l}(D)-\alpha(D)}{3}\right\rceil .
$$

Lemma 4 (see Reid [1] 1985 and Song [2] 1993). If D is a 2connected tournament with at least 6 vertices, then $D$ contains two complementary cycles of length $t$ and $|V(D)|-t$ for all $3 \leq$ $t \leq|V(D)| / 2$, unless it is isomorphic to the tournament $T_{7}$.

Lemma 5 (see Bondy [11] 1976). Each strong c-partite tournament with $c \geq 3$ contains an $m$-cycle for each $m \in\{3,4, \ldots, c\}$.

Lemma 6 (see Yeo [12] 1999). Let D be a c-partite tournament with the partite sets $V_{1}, V_{2}, \ldots, V_{c}$ such that $k=\left|V_{1}\right| \leq\left|V_{2}\right| \leq$ $\cdots \leq\left|V_{c}\right|=k+i$ for some $i \in\{0,1\}$. If

$$
i_{l}(D) \leq \frac{|V(D)|-\left|V_{c-1}\right|-2\left|V_{c}\right|+2}{2},
$$

then $D$ is Hamiltonian.
Lemma 7 (see Volkmann [5] 2004). Let D be a regular 3partite tournament with $|V(D)| \geq 6$. Then $D$ contains two complementary cycles of length 3 and $|V(D)|-3$, unless $D$ is isomorphic to $D_{3,2}$.

Lemma 8 (see Volkmann [4] 2005). Let $D$ be a regular $c$ partite tournament with $c \geq 4$ and $|V(D)| \geq 6$. Then $D$ contains two complementary cycles of length 3 and $|V(D)|-3$, unless $D$ is isomorphic to $T_{7}, D_{4,2}$, or $D_{4,2}^{*}$.

Lemma 9 (see Tewes [13] 2002). If $D$ is a multipartite tournament, then

$$
\begin{aligned}
\delta(D) & \geq \frac{|V(D)|-i_{l}(D)-\alpha(D)}{2} \\
& \geq \frac{|V(D)|-i_{g}(D)-\alpha(D)}{2} .
\end{aligned}
$$

Lemma 10 (see Yeo [12] 1999 and Gutin and Yeo [14] 2000). A digraph $D$ has no cycle factor if and only if its vertex set $V(D)$ can be partitioned into four subsets $Y, Z, R_{1}$, and $R_{2}$ such that

$$
\begin{aligned}
& R_{1} \Longrightarrow Y, \\
&\left(R_{1} \cup Y\right) \Longrightarrow R_{2}, \\
&|Y|>|Z|,
\end{aligned}
$$

where $Y$ is an independent set.

Lemma 11 (see Volkmann and Winzen [15] 2004). Let $V_{1}, V_{2}, \ldots, V_{c}$ be the partite sets of a c-partite tournament $D$ with no cycle factor such that $\left|V_{1}\right| \leq\left|V_{2}\right| \leq \cdots \leq\left|V_{c}\right|$. According to Lemma 10, the vertex set $V(D)$ can be partitioned into subsets $Y, Z, R_{1}$ and $R_{2}$ satisfying (4) such that $|Z|+k+1 \leq$ $|Y| \leq\left|V_{c}\right|-t$ with integers $k, t \geq 0$. Let $V_{i}$ be the partite set with the property that $Y \subseteq V_{i}$. If $Q=V(D)-Z-V_{i}, Q_{1}=Q \cap R_{1}$, and $Q_{2}=Q \cap R_{2}$, then

$$
\begin{aligned}
& i_{l}(D) \geq|V(D)|-3\left|V_{c}\right|+2 t+2 k+2, \\
& i_{g}(D) \geq \frac{|V(D)|-\left|V_{c-1}\right|-2\left|V_{c}\right|+3 k+3}{2},
\end{aligned}
$$

if $Q_{1}=\emptyset$ or $Q_{2}=\emptyset$ case part (1) of lemma and

$$
i_{g}(D) \geq i_{l}(D) \geq \frac{|V(D)|-\left|V_{c-1}\right|-2\left|V_{c}\right|+3 k+3+t}{2},
$$

if $Q_{1} \neq \emptyset$ and $Q_{2} \neq \emptyset$ case part (2) of lemma.

\section{Main Result}

Theorem 12. Let $D$ be a locally almost regular c-partite tournament with $c \geq 3$ and $|\gamma(D)| \leq 3$ such that all partite sets have the same cardinality, and let $C_{3}$ be a 3-cycle of $D$. We prove that if $D-V\left(C_{3}\right)$ has no cycle factor, then according to Lemma 10, the vertex set $V(D)$ can be partitioned into subsets $Y, Z, R_{1}$ and $R_{2}$ satisfying (4) such that $R_{1} \Rightarrow Y,\left(R_{1} \cup Y\right) \Rightarrow R_{2}$ and $|Y|>|Z|$, where $Y$ is an independent set. If $|Z \cup Y| \leq$ 4 , then $D$ contains a pair of disjoint cycles of length 3 and $|V(D)|-3$, unless $D$ is isomorphic to $T_{7}, D_{4,2}, D_{4,2}^{*}$, or $D_{3,2}$. 
Proof. First of all notice that the condition that all partite sets have the same cardinality shows that $i_{l}(D)=i_{g}(D)$. Let $V_{1}, V_{2}, \ldots, V_{c}$ be the partite sets of a locally almost regular $c$ partite tournament $D$ such that $\left|V_{1}\right|=\left|V_{2}\right|=\cdots=\left|V_{c}\right|=r$. If $|V(D)|-r$ is even, then it is easy to say that $D$ is regular. Thus, if $c=3$, then $D$ is a regular 3-partite tournament, and by Lemma 7, $D$ contains two complementary cycles of length 3 and $|V(D)|-3$, unless $D$ is isomorphic to $D_{3,2}$.

According to Lemma 3, we have

$$
\kappa(D) \geq\left\lceil\frac{|V(D)|-2 i_{l}(D)-\alpha(D)}{3}\right\rceil .
$$

If $c \geq 7$ and $r=1$, which means that $D$ is a tournament, then (7) yields $\kappa(D) \geq 2$. In addition, if $c=6$ and $r=1$, then it can be shown that $\kappa(D) \geq 2$ too. Hence Lemma 4 implies that $D$ contains two complementary cycles of length 3 and $|V(D)|-3$, unless $D$ is isomorphic to $T_{7}$.

Therefore we only need to consider the case $c \geq 4$ and $2 \leq r \leq 3$. In view of Lemma $3, \kappa(D) \geq 2$ and thus Lemma 5 ensures the existence of a 3 -cycle $C_{3}$ in $D$. If we define in the case $r \geq 2$ the $c$-partite tournament $H$ by $H=D-V\left(C_{3}\right)$, then $i_{l}(H) \leq 4$, and $|V(H)|=c r-3$. If $V_{1}^{\prime}, V_{2}^{\prime}, \ldots, V_{c}^{\prime}$ are the partite sets of $H$ such that $\left|V_{1}^{\prime}\right| \leq\left|V_{2}^{\prime}\right| \leq \cdots \leq\left|V_{c}^{\prime}\right|$, then $\left|V_{1}^{\prime}\right|=r-1$ and $\left|V_{c}^{\prime}\right|=r$. With exception of the cases $c=4$ and $r=2,3, c=5$ and $r=2,3, c=6$ and $r=2$, and $c=7$ and $r=2$, this leads to

$$
i_{l}(H) \leq 4 \leq \frac{|V(H)|-\left|V_{c-1}^{\prime}\right|-2\left|V_{c}^{\prime}\right|+2}{2} .
$$

Applying Lemma 6, we conclude that $H$ has a Hamiltonian cycle $C$, and we obtain the desired result that $V(D)=V\left(C_{3}\right) \cup$ $V(C)$. Since $D$ is a regular $c$-partite tournament for $c=4$ and $r=2, c=5$ and $r=2,3, c=6$ and $r=2$, and $c=7$ and $r=2$, by Lemma $8, D$ contains two complementary cycles of length 3 and $|V(D)|-3$, unless $D$ is isomorphic to $D_{4,2}$, or $D_{4,2}^{*}$. Therefore, there only remain the cases $c=4$ and $r=3$.

Let $V_{1}=\left\{u_{1}, u_{2}, u_{3}\right\}, V_{2}=\left\{v_{1}, v_{2}, v_{3}\right\}, V_{3}=\left\{w_{1}, w_{2}, w_{3}\right\}$, $V_{4}=\left\{x_{1}, x_{2}, x_{3}\right\}$ be the partite sets of $D, C_{3}=u_{3} v_{3} w_{3} u_{3}$, and $H=D-V\left(C_{3}\right)$. Then $\alpha(D)=3$ and $4 \leq d_{D}^{+}(x), d_{D}^{-}(x) \leq$ 5 for each $x \in V(D)$. Then $i_{l}(H) \leq 4$, and $|V(H)|=9$. If $V_{1}^{\prime}, V_{2}^{\prime}, V_{3}^{\prime}, V_{4}^{\prime}$ are the partite sets of $H$ such that $\left|V_{1}^{\prime}\right| \leq\left|V_{2}^{\prime}\right| \leq$ $\left|V_{3}^{\prime}\right| \leq\left|V_{4}^{\prime}\right|$, then $\left|V_{1}^{\prime}\right|=\left|V_{2}^{\prime}\right|=\left|V_{3}^{\prime}\right|=2$ and $\left|V_{4}^{\prime}\right|=3$. Then by Lemma 10, the vertex set $V(H)$ can be partitioned into subsets $Y, Z, R_{1}, R_{2}$ such that $R_{1} \Rightarrow Y,\left(R_{1} \cup Y\right) \Rightarrow R_{2},|Y|>|Z|$, and $Y$ is an independent set. Let, without loss of generality, $\left|R_{1}\right| \leq$ $\left|R_{2}\right|$ (if $\left|R_{1}\right| \geq\left|R_{2}\right|$, then we consider $D^{-1}$ ). By Lemma 9, $\delta(H) \geq 1$.

Case 1. $Z=\emptyset$ and $|Y|=1,2,3$. Then $R_{1} \neq \emptyset$. Otherwise $d_{D}^{+}(x) \geq 6$ and $d_{D}^{-}(x) \leq 3$ for $x \in Y$ which contradicts $i_{l}(D) \leq$ 1. Consider $\left|R_{1}\right| \neq 1,2$ for $\delta(H) \geq 1$. So $3 \leq\left|R_{1}\right| \leq 4$.

Assume that $|Y|=1$. We claim that $\left|R_{1}\right| \neq 3$, or else since $d_{H}^{+}(x), d_{H}^{-}(x) \geq 1, x \in V(H), R_{1}$ induces a 3 -cycle, and thus there is a vertex $y \in R_{1}$ such that $\left|V(y) \cap\left(Y \cup R_{2}\right)\right| \leq 1$. It follows that $d_{D}^{+}(y) \geq 6$ and $d_{D}^{-}(y) \leq 3$, a contradiction to $i_{l}(D) \leq 1$. Assume that $\left|R_{1}\right|=4$. If $R_{1}$ is bipartite, then since $\delta(H) \geq 1$, each partite set of $R_{1}$ consists of two vertices.
Hence, $R_{1}$ induces a 4-cycle and thus there is a vertex $y \in R_{1}$ such that $\left|V(y) \cap\left(Y \cup R_{2}\right)\right| \leq 1$. It follows that $d_{D}^{+}(y) \geq 6$ and $d_{D}^{-}(y) \leq 3$, a contradiction to $i_{l}(D) \leq 1$. If $R_{1}$ is 3 -partite, then there exists a vertex $y \in R_{1}$ such that $\left|V(y) \cap\left(Y \cup R_{2}\right)\right| \leq 2$ and $d_{R_{1}}^{+}(y) \geq 2$ or $\left|V(y) \cap\left(Y \cup R_{2}\right)\right| \leq 1$ and $d_{R_{1}}^{+}(y) \geq 1$. This leads to $d_{D}^{+}(y) \geq 6$ and $d_{D}^{-}(y) \leq 3$, a contradiction to $i_{l}(D) \leq 1$. If $R_{1}$ is 4-partite, then $y \in R_{1}$ such that $\left|V(y) \cap\left(Y \cup R_{2}\right)\right| \leq 1$ and $d_{R_{1}}^{+}(y) \geq 2$; then $d_{D}^{+}(y) \geq 6$ and $d_{D}^{-}(y) \leq 3$, a contradiction to $i_{l}(D) \leq 1$.

If $|Y|=2$, then $\left|R_{1}\right| \leq 3$. Note that $\delta(H) \geq 1, R_{1}$ induces a 3-cycle. So there is a vertex $y \in R_{1}$ such that $d_{D}^{+}(y) \geq 6$ and $d_{D}^{-}(y) \leq 3$, a contradiction to $i_{l}(D) \leq 1$.

If $|Y|=3$, then replacing $k$ by 2 and $t$ by 0 in Lemma 11 leads us to $i_{l}(H) \geq 5$, a contradiction to $i_{l}(H) \leq 4$.

Case 2. One has $|Z|=1$ and $|Y|=2$. We discuss this case by the value of $\left|R_{1}\right|$, where $\left|R_{1}\right|=0,1,2,3$.

Case 2.1. Assume that $\left|R_{1}\right|=0$; that is, $R_{1}=\emptyset$.

Note that $Z \rightarrow Y$ since $\delta(H) \geq 1$. If $Y \subset V_{i}$, for some $i \epsilon$ $\{1,2,3\}$, then $R_{2} \cap V_{i}=\emptyset$. Then $d_{D}^{+}(y) \geq 6$ and $d_{D}^{-}(y) \leq 3$ for $y \in Y$, a contradiction. Therefore, $Y \subset V_{4}$. Assume, without loss of generality, that $Y=\left\{x_{1}, x_{2}\right\}, x_{3} \in R_{2}$, and $Z=\left\{u_{1}\right\}$. Since $i_{l}(D) \leq 1, V\left(C_{3}\right) \rightarrow Y$, and there exists one vertex in $\left\{v_{1}, v_{2}, w_{1}, w_{2}\right\}$, say, $v_{1}$ such that $v_{1} \rightarrow u_{1}$.

Case 2.1.1. Assume that $H\left[\left\{u_{2}, v_{2}, w_{1}, w_{2}, x_{3}\right\}\right]$ has a Hamiltonian path.

(1) Assume that there is a Hamiltonian path which does not end at $x_{3}$ or begin at $x_{3}$, say, $p_{1} p_{2} p_{3} p_{4} p_{5}$ with $x_{3} \notin\left\{p_{1}, p_{5}\right\}$ in $H\left[\left\{u_{2}, v_{2}, w_{1}, w_{2}, x_{3}\right\}\right]$. If $p_{5}$ does not dominate $p_{1}$, then $p_{5}$ has at least one positive neighbor $x$ in $C_{3}$, and there are the complementary cycles $x_{1} v_{1} u_{1} x_{1}$ and $x_{2} p_{1} p_{2} p_{3} p_{4} p_{5} x x^{+} x^{++} x_{2}$. If $p_{5} \rightarrow p_{1}$, then there exist at least two arcs from $\left\{p_{1}, p_{2}, p_{3}, p_{4}, p_{5}\right\}$ to $C_{3}$. Let, without loss of generality, $p_{i} \rightarrow x$ with $p_{i}^{+} \neq x_{3}$ and $x \in$ $V\left(C_{3}\right)$. This leads to the complementary cycles $x_{1} v_{1} u_{1} x_{1}$ and $x_{2} p_{i+1} p_{i+2} \cdots p_{i} x x^{+} x^{++} x_{2}$, all indices are taken modulo 5 .

(2) If there is a Hamiltonian path ending at $x_{3}$ in $H\left[\left\{u_{2}, v_{2}, w_{1}, w_{2}, x_{3}\right\}\right]$, say, $p_{1} p_{2} p_{3} p_{4} p_{5}$ with $p_{5}=x_{3}$, then we need only to consider $\left\{p_{1}, p_{2}, p_{3}\right\} \rightarrow p_{5}$. The other cases are easily reduced to (1). Thus $p_{5}$ has at least one positive neighbor in $C_{3}$, say, $x$. This leads to the complementary cycles $x_{1} v_{1} u_{1} x_{1}$ and $x_{2} p_{1} p_{2} p_{3} p_{4} p_{5} x x^{+} x^{++} x_{2}$.

(3) If there is a Hamiltonian path beginning at $x_{3}$ in $H\left[\left\{u_{2}, v_{2}, w_{1}, w_{2}, x_{3}\right\}\right]$, say, $p_{1} p_{2} p_{3} p_{4} p_{5}$ with $p_{1}=x_{3}$, then we need only to consider $p_{1} \rightarrow\left\{p_{2}, p_{3}, p_{4}, p_{5}\right\}$. The other cases are easily reduced to (1) or (2).

Assume that $p_{1} \rightarrow v_{1}$. Then $\left(V\left(C_{3}\right) \cup\left\{u_{1}\right\}\right) \rightarrow p_{1}$, and we obtain a 3 -cycle $p_{1} v_{1} u_{1} p_{1}$. Next we are to find its complementary cycle. Since $i_{l}(D) \leq 1, p_{5}$ dominates at least one vertex $x$ of $C_{3}$, and there exists at least one vertex of $\left\{p_{2}, p_{3}, p_{4}\right\}$, say, $p_{i}$, such that $p_{i} \rightarrow y$ with $i \in\{2,3,4\}$, $y \in V\left(C_{3}\right)$ and $x \neq y$. This leads to the complementary cycles $C_{1}=p_{1} v_{1} u_{1} p_{1}$ and $C_{2}=x_{1} p_{2} \cdots p_{i} y y^{+} x_{2} p_{i+1} \cdots p_{5} x x_{1}$ if $y=x^{+}$or $C_{2}=x_{1} p_{2} \cdots p_{i} y x_{2} p_{i+1} \cdots p_{5} x x^{+} x_{1}$ if $y=x^{-}$.

Now assume that $v_{1} \rightarrow p_{1}$. If there is a vertex $p_{i}$ of $\left\{p_{2}, p_{3}, p_{4}, p_{5}\right\}$ that dominates $u_{1}(i \in\{2,3,4,5\})$, then let $p_{i}$ take the place of $v_{1}$, the above case being reduced to (1) of 
Case 2.1.1. Therefore, we let $u_{1} \Rightarrow\left\{p_{2}, p_{3}, p_{4}, p_{5}\right\}$. Then we have $p_{1} \cup V\left(C_{3}\right) \Rightarrow u_{1}$.

If $u_{2} \notin\left\{p_{3}, p_{5}\right\}$ and $V\left(p_{3}\right) \neq V\left(p_{5}\right)$, then there exists an arc between $p_{3}$ and $p_{5}$. Let, without loss of generality, $p_{3} \rightarrow$ $p_{5}$. This leads to the contradiction $d_{D}^{-}\left(p_{5}\right) \geq 6$.

If $u_{2} \notin\left\{p_{3}, p_{5}\right\}$ and $V\left(p_{3}\right)=V\left(p_{5}\right)$, then $\left\{p_{3}, p_{5}\right\}=$ $\left\{w_{1}, w_{3}\right\}$ and $\left\{p_{3}, p_{5}\right\} \Rightarrow\left\{v_{1}\right\} \cup V\left(C_{3}\right)$ and $p_{5} \rightarrow p_{2}$. If $p_{2} \rightarrow p_{4}$, then $p_{4}=u_{2}, p_{2}=v_{2},\left\{p_{2}, p_{4}\right\} \Rightarrow\left\{v_{1}\right\} \cup V\left(C_{3}\right)$, and $v_{1} \Rightarrow V\left(C_{3}\right)$. This leads to the complementary cycles $p_{2} p_{4} p_{5} p_{2}$ and $x_{1} p_{3} u_{3} x_{2} v_{1} p_{1} v_{3} w_{3} u_{1} x_{1}$. If $p_{4} \rightarrow p_{2}$, then $p_{2}=u_{2}, p_{4}=v_{2},\left\{p_{2}, p_{4}\right\} \Rightarrow\left\{v_{1}\right\} \cup V\left(C_{3}\right)$, and $v_{1} \Rightarrow$ $V\left(C_{3}\right)$. This leads to the complementary cycles $p_{2} p_{3} p_{4} p_{2}$ and $x_{1} p_{5} u_{3} x_{2} v_{1} p_{1} v_{3} w_{3} u_{1} x_{1}$.

If $u_{2} \in\left\{p_{3}, p_{5}\right\}$, let, without loss of generality, $p_{5}=u_{2}$; then we have $V\left(p_{2}\right)=V\left(p_{4}\right)=V\left(w_{1}\right), p_{3}=v_{2}, p_{3} \rightarrow$ $p_{5}, p_{5} \rightarrow p_{2},\left\{p_{2}, p_{4}, p_{5}\right\} \rightarrow v_{1}$, and $\left\{v_{1}, p_{2}, p_{3}, p_{4}, p_{5}\right\} \Rightarrow$ $V\left(C_{3}\right)$. This leads to the complementary cycles $p_{2} p_{3} p_{5} p_{2}$ and $x_{1} v_{1} p_{1} u_{1} x_{2} p_{4} u_{3} v_{3} w_{3} x_{1}$.

Case 2.1.2. Now we assume that $H\left[\left\{u_{2}, v_{2}, w_{1}, w_{2}, x_{3}\right\}\right]$ has no Hamiltonian path. Then let, without loss of generality, $u_{2} \rightarrow$ $v_{2}$.

(1)Assume that $v_{2}$ dominates one vertex $\left\{w_{1}, w_{2}\right\}$, say, $v_{2} \rightarrow w_{2}$.

(a) If $w_{2} \rightarrow x_{3}$, then since $H\left[\left\{u_{2}, v_{2}, w_{1}, w_{2}, x_{3}\right\}\right]$ has no Hamiltonian path, $w_{1} \rightarrow x_{3},\left\{u_{2}, v_{2}\right\} \rightarrow\left\{w_{1}, x_{3}\right\}$, and $u_{2} \rightarrow$ $w_{2}$.

If there is one vertex of $\left\{w_{1}, w_{2}\right\}$ that dominates $u_{1}$, let, without loss of generality, $w_{1} \rightarrow u_{1}$; then let $w_{1}$ take the place of $v_{1}$, the case being reduced to (1) of Case 2.1.1. Therefore, let $u_{1} \rightarrow\left\{w_{1}, w_{2}\right\}$. Then we have $\left\{w_{1}, w_{2}\right\} \Rightarrow$ $\left(\left\{v_{1}\right\} \cup V\left(C_{3}\right)\right)$. Since $i_{l}(D) \leq 1, x_{3} \rightarrow u_{3}$ or $x_{3} \rightarrow v_{3}$. In the former case there are complementary cycles $w_{1} v_{1} u_{1} w_{1}$ and $x_{1} u_{2} x_{3} u_{3} x_{2} v_{2} w_{2} v_{3} w_{3} x_{1}$; in the latter case complementary cycles are $w_{1} v_{1} u_{1} w_{1}$ and $x_{1} u_{2} w_{2} u_{3} x_{2} v_{2} x_{3} v_{3} w_{3} x_{1}$.

(b) Assume that $x_{3} \rightarrow w_{2}$.

If $w_{1} \rightarrow u_{2}$, then since $H\left[\left\{u_{2}, v_{2}, w_{1}, w_{2}, x_{3}\right\}\right]$ has no Hamiltonian path, $\left\{w_{1}, u_{2}, v_{2}\right\} \rightarrow x_{3}$. This leads to the Hamiltonian path $w_{1} u_{2} v_{2} x_{3} w_{2}$, a contradiction.

If $u_{2} \rightarrow w_{1}$ and $w_{1} \rightarrow v_{2}$, then since $H\left[\left\{u_{2}, v_{2}, w_{1}, w_{2}\right.\right.$, $\left.\left.x_{3}\right\}\right]$ has no Hamiltonian path, $x_{3} \rightarrow\left\{w_{1}, u_{2}, v_{2}\right\}$. This leads to the Hamiltonian path $x_{3} u_{2} w_{1} v_{2} w_{2}$, a contradiction.

If $u_{2} \rightarrow w_{1}$ and $v_{2} \rightarrow w_{1}$, then since $H\left[\left\{u_{2}, v_{2}, w_{1}, w_{2}\right.\right.$, $\left.\left.x_{3}\right\}\right]$ has no Hamiltonian path, $x_{3} \rightarrow w_{1}, u_{2} \rightarrow w_{2}$, and $\left\{w_{1}, w_{2}\right\} \Rightarrow\left(\left\{v_{1}, u_{1}\right\} \cup V\left(C_{3}\right)\right)$. If $v_{2} \rightarrow x_{3}$ or $x_{3} \rightarrow v_{2}$ and $u_{2} \rightarrow x_{3}$, then let $w_{1}$ take the place of $v_{1}$, the case being reduced to (1) of Case 2.1.1. If $x_{3} \rightarrow v_{2}$ and $x_{3} \rightarrow u_{2}$, then let $w_{1}$ take the place of $v_{1}$, the case being reduced to (3) of Case 2.1.1.

(2) Assume that $\left\{w_{1}, w_{2}\right\} \rightarrow v_{2}$. Then we have $v_{2} \Rightarrow(Z \cup$ $\left.V\left(C_{3}\right) \cup\left\{x_{3}\right\}\right)$.

(a) If $u_{2}$ dominates one vertex of $\left\{w_{1}, w_{2}\right\}$, say, $w_{1}$, then since $H\left[\left\{u_{2}, v_{2}, w_{1}, w_{2}, x_{3}\right\}\right]$ has no Hamiltonian path, $\left\{w_{1}, w_{2}\right\} \rightarrow x_{3}$ and $u_{2} \rightarrow\left\{x_{3}, w_{2}\right\}$.

(i) If $x_{3} \rightarrow v_{1}$ and $v_{1}$ dominates one vertex of $\left\{w_{1}, w_{2}\right\}$, then let $v_{2}$ take the place of $v_{1}$, the case being reduced to (3) of Case 2.1.1.

(ii) If $x_{3} \rightarrow v_{1}$ and $\left\{w_{1}, w_{2}\right\} \rightarrow v_{1}$, then let $v_{2}$ take the place of $v_{1}$, the case being reduced to (2) of Case 2.1.1. (iii) Assume that $v_{1} \rightarrow x_{3}$; then we have $x_{3} \rightarrow(Z \cup$ $\left.V\left(C_{3}\right)\right)$

If $v_{3}$ dominates one vertex of $\left\{u_{2}, w_{1}, w_{2}\right\}$, say, $v_{3} \rightarrow$ $w_{1}$, then we obtain the complementary cycles $w_{1} x_{3} v_{3} w_{1}$ and $x_{1} v_{1} u_{1} x_{2} u_{2} w_{2} v_{2} w_{3} u_{3} x_{1}$.

If $\left\{u_{2}, w_{1}, w_{2}\right\} \rightarrow v_{3}$, then we have $v_{1} \Rightarrow\left(\left\{u_{2}\right\} \cup V\left(C_{3}\right)\right)$. Then let $v_{2}$ take the place of $v_{1}$, the case being reduced to (2) of Case 2.1.1.

(b) Assume that $\left\{w_{1}, w_{2}\right\} \rightarrow u_{2}$. Since $H\left[\left\{u_{2}, v_{2}, w_{1}, w_{2}\right.\right.$, $\left.x_{3}\right\}$ ] has no Hamiltonian path, $\left\{w_{1}, w_{2}\right\} \rightarrow x_{3}$.

(i) Assume that $x_{3} \rightarrow u_{2}$. Then $u_{2} \Rightarrow\left(V\left(C_{3}\right) \cup\left\{v_{1}\right\}\right)$.

If $x_{3} \rightarrow v_{1}$, then $v_{1}$ dominates at least one vertex of $\left\{w_{1}, w_{2}\right\}$; then let $v_{2}$ take the place of $v_{1}$, the case being reduced to (1) of Case 2.1.1.

If $v_{1} \rightarrow x_{3}$ and there exists one vertex of $\left\{w_{1}, w_{2}\right\}$ that dominates at least one vertex of $C_{3}$, say, $w_{1} \rightarrow v_{3}$, then we obtain the complementary cycles $C_{1}=x_{3} u_{2} v_{1} x_{3}$ and $C_{2}=$ $x_{1} w_{2} v_{2} u_{1} x_{2} w_{1} v_{3} w_{3} u_{3} x_{1}$.

If $v_{1} \rightarrow x_{3}$ and $V\left(C_{3}\right) \Rightarrow\left\{w_{1}, w_{2}\right\}$, then $R_{2} \backslash\left\{w_{1}\right.$, $\left.w_{2}\right\} \Rightarrow\left\{u_{3}, v_{3}\right\}$. This leads to the complementary cycles $C_{1}=$ $w_{1} v_{2} u_{3} w_{1}$ and $C_{2}=x_{1} v_{1} u_{1} x_{2} w_{2} x_{3} u_{2} v_{3} w_{3} x_{1}$.

(ii) Assume that $u_{2} \rightarrow x_{3}$.

If $x_{3} \rightarrow v_{1}$ and $v_{1}$ dominates one vertex of $\left\{w_{1}, w_{2}\right\}$, then let $v_{2}$ take the place of $v_{1}$, the case being reduced to (1) of Case 2.1.1.

Assume that $\left\{w_{1}, w_{2}, x_{3}\right\} \rightarrow v_{1}$. Then we have $v_{1} \Rightarrow$ $\left(V\left(C_{3}\right) \cup\left\{u_{2}\right\}\right)$ and $u_{2} \Rightarrow V\left(C_{3}\right)$. If there exists one vertex of $\left\{w_{1}, w_{2}\right\}$ that dominates $u_{1}$, say, $w_{1} \rightarrow u_{1}$, then let $w_{1}$ take the place of $v_{1}$, the case being reduced to (2) of Case 2.1.1. Therefore, let $u_{1} \rightarrow\left\{w_{1}, w_{2}\right\}$. This leads to the complementary cycles $C_{1}=w_{1} v_{1} u_{1} w_{1}$ and $C_{2}=x_{1} v_{2} u_{3} x_{2} w_{2} u_{2} x_{3} v_{3} w_{3} x_{1}$.

Now assume that $v_{1} \rightarrow x_{3}$. Then we have $x_{3} \rightarrow\left(\left\{u_{1}\right\} \cup\right.$ $\left.V\left(C_{3}\right)\right)$.

Let $v_{1}$ dominate one vertex of $\left\{w_{1}, w_{2}\right\}$, say, $v_{1} \rightarrow w_{1}$.

If $w_{2} \rightarrow u_{1}$, then we have the complementary cycles $C_{1}=$ $x_{1} w_{2} u_{1} x_{1}$ and $C_{2}=x_{2} v_{1} w_{1} u_{2} v_{2} x_{3} u_{3} v_{3} w_{3} x_{2}$.

If $u_{1} \rightarrow w_{2}$, then since $i_{l}(D) \leq 1, u_{2}$ dominates at least one vertex of $C_{3}$. This leads to the complementary cycles $C_{1}=$ $w_{2} v_{2} u_{1} w_{2}$ and $C_{2}=x_{1} w_{1} u_{2} v_{3} w_{3} x_{2} v_{1} x_{3} u_{3} x_{1}$ if $u_{2} \rightarrow v_{3}$ or $C_{2}=x_{1} w_{1} u_{2} w_{3} x_{2} v_{1} x_{3} u_{3} v_{3} x_{1}$ if $u_{2} \rightarrow w_{3}$.

Now let $\left\{w_{1}, w_{2}\right\} \rightarrow v_{1}$.

If $u_{1}$ dominates one vertex of $\left\{w_{1}, w_{2}\right\}$, say, $u_{1} \rightarrow w_{1}$, then we obtain the complementary cycles $C_{1}=v_{1} u_{1} w_{1} v_{1}$ and $C_{2}=$ $x_{1} w_{2} v_{2} u_{3} x_{2} u_{2} x_{3} v_{3} w_{3} x_{1}$.

If $\left\{w_{1}, w_{2}\right\} \rightarrow u_{1}$, then we have $u_{1} \Rightarrow V\left(C_{3}\right)$ and $V\left(C_{3}\right) \Rightarrow\left\{w_{1}, w_{2}\right\}$. This leads to the complementary cycles $C_{1}=w_{1} u_{1} v_{3} w_{1}$ and $C_{2}=x_{1} w_{2} u_{2} v_{2} w_{3} x_{2} v_{1} x_{3} u_{3} x_{1}$.

Case 2.2. Assume that $\left|R_{1}\right|=1$. If $R_{1} \cap V_{4}=\emptyset$, then $\mid V(x) \cap$ $\left(Y \cup R_{2}\right) \mid=1$ for $x \in R_{1}$. Therefore, we have $d_{D}^{+}(x) \geq 6$ and $d_{D}^{-}(x) \leq 3$ for $x \in R_{1}$, a contradiction. Hence, let, without loss of generality, $R_{1}=\left\{x_{3}\right\}$. If $Y=\left\{x_{1}, x_{2}\right\}$, then the case is equivalent to the case of " $|Y|=2, x_{3} \in R_{2}$, and $x_{3} \rightarrow$ $R_{2} \backslash\left\{x_{3}\right\}$," which has been proved in (3) of Case 2.1.1 and Case 2.1.2. If $Z \cap V_{4} \neq \emptyset$, then $d_{D}^{+}\left(x_{3}\right) \geq 6$, a contradiction. So, let, without loss of generality, $Y=\left\{u_{1}, u_{2}\right\}$ and $Z=\left\{v_{1}\right\}$. Then $\left(V\left(C_{3}\right) \cup Z\right) \Rightarrow R_{1}$ and $\left(V\left(C_{3}\right) \cup Z\right) \Rightarrow Y$.

(1) Assume that there is an arc from $\left\{x_{1}, x_{2}\right\}$ to $v_{1}$, say, $x_{1} \rightarrow v_{1}$. Then we have $C_{1}=x_{1} v_{1} u_{1} x_{1}$. 
(a) Assume that $H\left[\left\{w_{1}, w_{2}, v_{2}, x_{2}\right\}\right]$ has a Hamiltonian path.

If there is a Hamiltonian path which does not end at $x_{2}$ or begin at $x_{2}$, let, without loss of generality, $p_{1} p_{2} p_{3} p_{4}$ with $x_{2} \notin$ $\left\{p_{1}, p_{4}\right\}$ be a Hamiltonian path in $H\left[\left\{w_{1}, w_{2}, v_{2}, x_{2}\right\}\right]$; then $p_{4}$ has at least one positive neighbor in $C_{3}$, say, $p_{4} \rightarrow u_{3}$, and we have the complementary cycle $C_{2}=w_{3} x_{3} u_{2} p_{1} p_{2} p_{3} p_{4} u_{3} v_{3} w_{3}$.

If there is a Hamiltonian path ending at $x_{2}$, let, without loss of generality, $p_{1} p_{2} p_{3} p_{4}$ with $p_{4}=x_{2}$ be a Hamiltonian path in $H\left[\left\{w_{1}, w_{2}, v_{2}, x_{2}\right\}\right]$; then we need only to consider $\left\{p_{1}, p_{2}, p_{3}\right\} \rightarrow p_{4}$. The other cases are easily reduced to the above case which does not end at $x_{2}$. Thus $p_{4} \rightarrow$ $\left(\left\{v_{1}\right\} \cup V\left(C_{3}\right)\right)$ and there is the complementary cycle $C_{2}=$ $u_{3} x_{3} u_{2} p_{1} p_{2} p_{3} p_{4} v_{3} w_{3} u_{3}$.

If there is a Hamiltonian path beginning at $x_{2}$, let, without loss of generality, $p_{1} p_{2} p_{3} p_{4}$ with $p_{1}=x_{2}$ be a Hamiltonian path in $H\left[\left\{w_{1}, w_{2}, v_{2}, x_{2}\right\}\right]$; then we need only to consider $p_{1} \rightarrow\left\{p_{2}, p_{3}, p_{4}\right\}$. The other cases are easily reduced to the above case which does not begin at $x_{2}$. Thus $p_{4} \Rightarrow$ $\left(\left\{v_{1}, x_{1}\right\} \cup V\left(C_{3}\right)\right)$ and we have the complementary cycle $C_{2}=$ $x_{3} u_{2} p_{1} p_{2} p_{3} p_{4} u_{3} v_{3} w_{3} x_{3}$.

(b) Now we assume that $H\left[\left\{w_{1}, w_{2}, v_{2}, x_{2}\right\}\right]$ has no Hamiltonian path. Let, without loss of generality, $v_{2} \rightarrow w_{2}$.

If $w_{2} \rightarrow x_{2}$, then since $H\left[\left\{w_{1}, w_{2}, v_{2}, x_{2}\right\}\right]$ has no Hamiltonian path, $w_{1} \rightarrow x_{2}, v_{2} \rightarrow x_{2}$, and $v_{2} \rightarrow w_{1}$. Then we have $x_{2} \rightarrow V\left(C_{3}\right)$ and $w_{1}$ dominates at least one vertex of $C_{3}$, say, $w_{1} \rightarrow u_{3}$. This leads to the complementary cycle $C_{2}=u_{3} x_{3} v_{2} w_{2} x_{2} v_{3} w_{3} u_{2} w_{1} u_{3}$.

If $x_{2} \rightarrow w_{2}$ and $v_{2} \rightarrow x_{2}$, then $v_{2} \rightarrow w_{1}$ and $x_{2} \rightarrow w_{1}$. This leads to $\left\{w_{1}, w_{2}\right\} \Rightarrow V\left(C_{3}\right)$ and the complementary cycle $C_{2}=u_{3} x_{3} v_{2} x_{2} w_{2} v_{3} w_{3} u_{2} w_{1} u_{3}$.

If $x_{2} \rightarrow w_{2}$ and $x_{2} \rightarrow v_{2}$, then $\left\{x_{2}, v_{2}\right\} \rightarrow w_{1}$ and $\left\{w_{1}, w_{2}\right\} \Rightarrow V\left(C_{3}\right)$. This leads to the complementary cycle $C_{2}=u_{3} x_{3} v_{2} w_{1} v_{3} w_{3} u_{2} x_{2} w_{2} u_{3}$.

(2) Assume that $v_{1} \rightarrow\left\{x_{1}, x_{2}\right\}$. Then we have $\left(\left\{w_{1}, w_{2}\right\} \cup\right.$ $\left.V\left(C_{3}\right)\right) \Rightarrow v_{1}, R_{2} \Rightarrow w_{3}$, and $C_{1}=w_{1} v_{1} u_{1} w_{1}$.

(a) Assume that $H\left[\left\{v_{2}, w_{2}, x_{1}, x_{2}\right\}\right]$ has a Hamiltonian path. Then, since $d_{D}^{+}(x) \leq 5$, there exists a Hamiltonian ending at $x_{1}$ or $x_{2}$; let, without loss of generality, $p_{1} p_{2} p_{3} p_{4}$ with $p_{4}=x_{1}$ be a Hamiltonian path $H\left[\left\{v_{2}, w_{2}, x_{1}, x_{2}\right\}\right]$. This leads to the complementary cycle $C_{2}=w_{3} u_{3} v_{3} x_{3} u_{2} p_{1} p_{2} p_{3} p_{4} w_{3}$.

(b) Assume that $H\left[\left\{v_{2}, w_{2}, x_{1}, x_{2}\right\}\right]$ has no Hamiltonian path.

If $v_{2} \rightarrow w_{2}$, then $w_{2} \rightarrow x_{1}$ or $w_{2} \rightarrow x_{2}$, say, $w_{2} \rightarrow x_{1}$. Since $H\left[\left\{v_{2}, w_{2}, x_{1}, x_{2}\right\}\right]$ has no Hamiltonian path, $\left\{v_{2}, w_{2}\right\} \rightarrow$ $x_{2}$ and $v_{2} \rightarrow x_{1}$. This leads to $\left\{x_{1}, x_{2}\right\} \rightarrow V\left(C_{3}\right)$ and we obtain the complementary cycle $C_{2}=u_{3} x_{3} v_{2} w_{2} x_{1} v_{3} w_{3} u_{2} x_{2} u_{3}$.

If $w_{2} \rightarrow v_{2}$, then $v_{2} \rightarrow x_{1}$ or $v_{2} \rightarrow x_{2}$, say, $v_{2} \rightarrow x_{1}$. Since $H\left[\left\{v_{2}, w_{2}, x_{1}, x_{2}\right\}\right]$ has no Hamiltonian path, $\left\{v_{2}, w_{2}\right\} \rightarrow x_{2}$ and $w_{2} \rightarrow x_{1}$. This leads to $\left\{x_{1}, x_{2}\right\} \rightarrow V\left(C_{3}\right)$ and we obtain the complementary cycle $C_{2}=u_{3} x_{3} w_{2} v_{2} x_{1} v_{3} w_{3} u_{2} x_{2} u_{3}$.

Case 2.3. Assume that $\left|R_{1}\right|=2$. If $R_{1} \cap V_{4}=\emptyset$, then there exists a vertex $x \in R_{1}$ such that $\left|V(x) \cap R_{1}\right|=2$ or $d_{R_{1}}^{+}(x)=1$. Each case leads to $d_{D}^{+}(x) \geq 6$ and $d_{D}^{-}(x) \leq 3$, a contradiction to $i_{l}(D) \leq 1$. Therefore, $R_{1} \cap V_{4} \neq \emptyset$. Let, without loss of generality, $x_{1} \in R_{1}$.
(1) Assume that $R_{1} \subset V_{4}$. Let, without loss of generality, $R_{1}=\left\{x_{1}, x_{2}\right\}$ and $Y=\left\{u_{1}, u_{2}\right\}$. Then $x_{3} \in R_{2}$. Let, without loss of generality, $Z=\left\{v_{1}\right\}$. Since $i_{l}(D) \leq 1,\left(Z \cup V\left(C_{3}\right)\right) \Rightarrow R_{1}$, $v_{2} \rightarrow w_{1}$, or $v_{2} \rightarrow w_{2}$, say, $v_{2} \rightarrow w_{2}$. Then we have $w_{2} \Rightarrow$ $\left(\left\{v_{1}, x_{3}\right\} \cup V\left(C_{3}\right)\right)$.

Assume that there is an arc from $Z$ to $Y$, say, $v_{1} \rightarrow u_{1}$.

If $w_{1} \rightarrow v_{2}$, then we have $v_{2} \Rightarrow\left(V\left(C_{3}\right) \cup\right.$ $\left.\left\{x_{3}\right\}\right)$ and $x_{3}$ dominates at least two vertices of $C_{3}$. This leads to the complementary cycles $C_{1}=u_{1} w_{2} v_{1} u_{1}$ and $C_{2}=u_{2} x_{3} u_{3} v_{3} x_{1} w_{1} v_{2} w_{3} x_{2} u_{2}$ if $x_{3} \rightarrow u_{3}$, or $C_{2}=$ $u_{2} x_{3} v_{3} x_{1} w_{1} v_{2} w_{3} u_{3} x_{2} u_{2}$ if $x_{3} \rightarrow v_{3}$.

If $v_{2} \rightarrow w_{1}$, then $w_{1} \Rightarrow\left(V\left(C_{3}\right) \cup\left\{x_{3}\right\}\right)$, and $x_{3}$ dominates at least two vertices of $C_{3}$. This leads to the complementary cycles $C_{1}=u_{1} w_{2} v_{1} u_{1}$ and $C_{2}=u_{2} x_{3} u_{3} x_{1} v_{2} w_{1} v_{3} w_{3} x_{2} u_{2}$ if $x_{3} \rightarrow u_{3}$, or $C_{2}=u_{2} x_{3} v_{3} x_{1} v_{2} w_{1} w_{3} u_{3} x_{2} u_{2}$ if $x_{3} \rightarrow v_{3}$.

Now assume that $Y \rightarrow Z$. Then we have $V\left(C_{3}\right) \rightarrow Y$, $R_{2} \Rightarrow w_{3}$, and $C_{1}=u_{1} v_{1} x_{1} u_{1}$.

If $w_{1} \rightarrow v_{2}$, then we have $v_{2} \Rightarrow\left(V\left(C_{3}\right) \cup\left\{w_{2}, x_{3}\right\}\right)$ and $w_{2} \rightarrow x_{3}$. This leads to the complementary cycle $C_{2}=$ $u_{2} w_{1} v_{2} w_{2} x_{3} w_{3} u_{3} v_{3} u_{2}$.

If $v_{2} \rightarrow w_{1}$, then $w_{1} \Rightarrow\left(V\left(C_{3}\right) \cup\left\{x_{3}\right\}\right)$ and we obtain the complementary cycle $C_{2}=u_{2} v_{2} w_{1} x_{3} w_{3} u_{3} x_{2} w_{2} v_{3} u_{2}$.

(2) Assume that $\left|R_{1} \cap V_{4}\right|=1$. Let, without loss of generality, $R_{1}=\left\{x_{1}, u_{1}\right\}$. Then $x_{1} \rightarrow u_{1}, u_{2} \in R_{2}$, and $\left(Z \cup V\left(C_{3}\right)\right) \Rightarrow R_{1}$. Since $i_{l}(D) \leq 1, Z \cap V_{4}=\emptyset$. Let, without loss of generality, $Z=\left\{v_{1}\right\}$.

(a) Assume that $Y \cap V_{4}=\emptyset$. Let, without loss of generality, $Y=\left\{w_{1}, w_{2}\right\}$. Then $R_{2}=\left\{x_{2}, x_{3}, u_{2}, v_{2}\right\}$.

(i) Assume that there is an arc from $Y$ to $Z$, say, $w_{1} \rightarrow v_{1}$. Then we have $C_{1}=w_{1} v_{1} x_{1} w_{1}$.

If $H\left[R_{2}\right]$ has a Hamiltonian path, then it ends at $x_{2}$ (or $\left.x_{3}\right)$. Let, without loss of generality, $p_{1} p_{2} p_{3} p_{4}$ with $p_{4} \in$ $\left\{x_{2}, x_{3}\right\}$ be a Hamiltonian path in $H\left[R_{2}\right]$. Then $p_{4}$ dominates at least two vertices of $C_{3}$. This leads to the complementary cycle $C_{2}=w_{2} p_{1} p_{2} p_{3} p_{4} u_{3} v_{3} w_{3} u_{1} w_{2}$ if $p_{4} \rightarrow u_{3}$ or $C_{2}=$ $w_{2} p_{1} p_{2} p_{3} p_{4} w_{3} u_{3} v_{3} u_{1} w_{2}$ if $p_{4} \rightarrow w_{3}$.

Now assume that $H\left[R_{2}\right]$ has no Hamiltonian path.

If $u_{2} \rightarrow v_{2}$, then $v_{2} \Rightarrow\left(\left\{x_{2}, x_{3}\right\} \cup V\left(C_{3}\right)\right)$. Since $H\left[R_{2}\right]$ has no Hamiltonian path, $u_{2} \rightarrow\left\{x_{2}, x_{3}\right\}$. Then we have $\left\{x_{2}, x_{3}\right\} \rightarrow\left(Z \cup V\left(C_{3}\right)\right)$. If $u_{3} \rightarrow w_{2}$, then we have the complementary cycle $C_{2}=u_{2} v_{2} x_{2} v_{3} u_{1} x_{3} w_{3} u_{3} w_{2} u_{2}$. If $w_{2} \rightarrow$ $u_{3}$, then $v_{3} \rightarrow w_{2}$ and we have the complementary cycle $C_{2}=u_{2} v_{2} x_{2} w_{3} u_{1} x_{3} u_{3} v_{3} w_{2} u_{2}$

If $v_{2} \rightarrow u_{2}$, then $u_{2}$ dominates one vertex of $\left\{x_{2}, x_{3}\right\}$, say, $u_{2} \rightarrow x_{2}$. Since $H\left[R_{2}\right]$ has no Hamiltonian path, $v_{2} \rightarrow$ $\left\{x_{2}, x_{3}\right\}$ and $u_{2} \rightarrow x_{3}$. Then we have $\left\{x_{2}, x_{3}\right\} \rightarrow\left(Z \cup V\left(C_{3}\right)\right)$. If $u_{3} \rightarrow w_{2}$, then we have the complementary cycle $C_{2}=$ $v_{2} u_{2} x_{2} v_{3} u_{1} x_{3} w_{3} u_{3} w_{2} v_{2}$. If $w_{2} \rightarrow u_{3}$, then $v_{3} \rightarrow w_{2}$ and we have the complementary cycle $C_{2}=v_{2} u_{2} x_{2} w_{3} u_{1} x_{3} u_{3} v_{3} w_{2} v_{2}$.

(ii) Assume that $Z \rightarrow Y$. Then there is an arc from $\left\{x_{2}, x_{3}\right\}$ to $Z$, say, $x_{2} \rightarrow v_{1}$. This leads to the 3 -cycle $C_{1}=w_{1} x_{2} v_{1} w_{1}$.

If $u_{2} \rightarrow v_{2}$, then $v_{2} \Rightarrow x_{3}$ and $x_{3}$ dominates at least two vertices of $C_{3}$. This leads to the complementary cycle $C_{2}=u_{2} v_{2} x_{3} u_{3} v_{3} w_{3} x_{1} u_{1} w_{2} u_{2}$ if $x_{3} \rightarrow u_{3}$ or $C_{2}=$ $u_{2} v_{2} x_{3} v_{3} w_{3} u_{3} x_{1} u_{1} w_{2} u_{2}$ if $x_{3} \rightarrow v_{3}$.

If $v_{2} \rightarrow u_{2}$ and $u_{2} \rightarrow x_{3}$, then $x_{3}$ dominates at least two vertices of $C_{3}$. This leads to the complementary cycle $C_{2}=v_{2} u_{2} x_{3} u_{3} v_{3} w_{3} x_{1} u_{1} w_{2} v_{2}$ if $x_{3} \rightarrow u_{3}$ or $C_{2}=$ $v_{2} u_{2} x_{3} v_{3} w_{3} u_{3} x_{1} u_{1} w_{2} v_{2}$ if $x_{3} \rightarrow v_{3}$. 
If $v_{2} \rightarrow u_{2}$ and $x_{3} \rightarrow u_{2}$, then $u_{2} \Rightarrow V\left(C_{3}\right)$. This leads to the complementary cycle $C_{2}=v_{2} x_{3} u_{2} v_{3} w_{3} u_{3} x_{1} u_{1} w_{2} v_{2}$ if $v_{2} \rightarrow x_{3}$ or $C_{2}=x_{3} v_{2} u_{2} v_{3} w_{3} u_{3} x_{1} u_{1} w_{2} v_{2}$ if $x_{3} \rightarrow v_{2}$.

(b) Assume that $Y \subset V_{4}$. Then let, without loss of generality, $Y=\left\{x_{2}, x_{3}\right\}$. Then we have $R_{2}=\left\{u_{2}, v_{2}, w_{1}, w_{2}\right\}$.

(i) Assume that there is an $\operatorname{arc}$ from $Z$ to $Y$, say, $v_{1} \rightarrow x_{2}$. Then there is an arc from $R_{2}$ to $Z$.

If there is an arc from $\left\{w_{1}, w_{2}\right\}$ to $Z$, say, $w_{1} \rightarrow v_{1}$, then we have $C_{1}=x_{2} w_{1} v_{1} x_{2}$. Let, without loss of generality, $v_{2} \rightarrow w_{2}$. Then $w_{2} \Rightarrow\left(\left\{u_{2}\right\} \cup V\left(C_{3}\right)\right)$ and $u_{2}$ dominates at least one vertex of $C_{3}$. This leads to the complementary cycle $C_{2}=v_{2} w_{2} u_{2} v_{3} w_{3} u_{3} x_{1} u_{1} x_{3} v_{2}$ if $u_{2} \rightarrow v_{3}$ or $C_{2}=$ $v_{2} w_{2} u_{2} w_{3} u_{3} v_{3} x_{1} u_{1} x_{3} v_{2}$ if $u_{2} \rightarrow w_{3}$.

Otherwise, $u_{2} \rightarrow v_{1}$. Then we have 3-cycle $C_{1}=$ $x_{2} u_{2} v_{1} x_{2}$. Let, without loss of generality, $v_{2} \rightarrow w_{2}$. Then $w_{2} \Rightarrow\left(Z \cup V\left(C_{3}\right)\right)$.

If $w_{1} \rightarrow v_{2}$, then we have the complementary cycle $C_{2}=$ $x_{3} w_{1} v_{2} w_{2} u_{3} v_{3} w_{3} x_{1} u_{1} x_{3}$.

If $v_{2} \rightarrow w_{1}$, then $w_{1} \Rightarrow Z \cup V\left(C_{3}\right)$ and we have the complementary cycle $C_{2}=v_{2} w_{2} u_{3} x_{1} w_{1} v_{3} w_{3} u_{1} x_{3} v_{2}$.

(ii) Assume that $Y \rightarrow Z$. Then $V\left(C_{3}\right) \rightarrow Y$ and we have 3 -cycle $C_{1}=x_{2} v_{1} u_{1} x_{2}$.

If $w_{2} \rightarrow v_{2}$, then $v_{2} \Rightarrow\left\{w_{1}, u_{2}\right\} \cup V\left(C_{3}\right), w_{1} \Rightarrow\left\{u_{2}\right\} \cup$ $V\left(C_{3}\right)$, and $u_{2} \Rightarrow V\left(C_{3}\right)$. This leads to the complementary cycle $C_{2}=w_{2} v_{2} u_{3} x_{1} w_{1} u_{2} v_{3} w_{3} x_{3} w_{2}$.

Now assume that $v_{2} \rightarrow w_{2}$. Then $w_{2} \Rightarrow\left\{u_{2}\right\} \cup V\left(C_{3}\right)$.

If $u_{2} \rightarrow w_{1}$, then $w_{1} \Rightarrow V\left(C_{3}\right)$ and we have the complementary cycle $C_{2}=u_{2} w_{1} v_{3} w_{3} x_{1} v_{2} w_{2} u_{3} x_{3} u_{2}$.

If $w_{1} \rightarrow u_{2}$, then $u_{2} \Rightarrow V\left(C_{3}\right)$ and we have the complementary cycle $C_{2}=w_{1} u_{2} v_{3} w_{3} x_{1} v_{2} w_{2} u_{3} x_{3} w_{1}$.

Case 2.4. Assume that $\left|R_{1}\right|=3$.

Let $R_{1} \cap V_{4}=\emptyset$. If $R_{2} \cap V_{4} \neq \emptyset$, then there exists one vertex $x \in R_{1}$ such that $d_{D}^{+}(x) \geq 6$, a contradiction to $i_{l}(D) \leq 1$. Therefore, $Z \cup Y=\left\{x_{1}, x_{2}, x_{3}\right\}$. Let, without loss of generality, $Z=\left\{x_{1}\right\}, Y=\left\{x_{2}, x_{3}\right\}$. Since $i_{l}(D) \leq 1$, let, without loss of generality, the set $R_{1}$ induce the 3-cycle $u_{1} v_{1} w_{1} u_{1}$, and the set $R_{2}$ induces the 3 -cycle $u_{2} v_{2} w_{2} u_{2}$ such that $R_{2} \Rightarrow Z \Rightarrow$ $R_{1}$. In addition, $i_{l}(D) \leq 1$ implies $R_{2} \Rightarrow V\left(C_{3}\right) \Rightarrow R_{1}$. It is evident that $u_{2} x_{1} v_{1} u_{2}$ and $x_{2} v_{2} u_{3} w_{1} x_{3} w_{2} v_{3} w_{3} u_{1} x_{2}$ are complementary cycles in $D$.

If $R_{1}=V_{4}$, then there exist two vertices $x, y \in R_{2}$ such that $(V(x) \cup V(y)) \cap\left(Y \cup R_{1}\right)=\emptyset$. Since there is an arc between $x$ and $y, d_{D}^{+}(x) \geq 6$ or $d_{D}^{+}(y) \geq 6$, a contradiction.

Therefore, $R_{1} \cap V_{4} \neq \emptyset$ and $R_{1} \neq V_{4}$. Thus $\left|R_{1} \cap V_{4}\right|=1$ or 2.

(1) Assume that $\left|R_{1} \cap V_{4}\right|=1$ first. Let, without loss of generality, $\left\{x_{1}, u_{1}\right\} \subset R_{1}$.

(a) If $u_{2} \in R_{1}$, then $x_{1} \rightarrow\left\{u_{1}, u_{2}\right\}$ and $\left\{x_{2}, x_{3}\right\} \subset\left(Y \cup R_{2}\right)$.

(i) If $Y=\left\{x_{2}, x_{3}\right\}$, then there exists one vertex $x \in R_{2}$ such that $d_{D}^{-}(x) \geq 6$, a contradiction.

(ii) If, without loss of generality, $Y=\left\{w_{1}, w_{2}\right\}$ and $Z=$ $\left\{v_{1}\right\}$, then $R_{2}=\left\{x_{2}, x_{3}, v_{2}\right\}$. Then we have $v_{2} \rightarrow\left\{x_{2}, x_{3}\right\}$, $R_{2} \Rightarrow\left(V\left(C_{3}\right) \cup Z\right)$, and $\left(V\left(C_{3}\right) \cup Z\right) \Rightarrow R_{1}$. This leads to the complementary cycles $C_{1}=x_{2} v_{2} u_{1} x_{2}$ and $C_{2}=$ $w_{1} x_{3} u_{3} v_{3} u_{2} w_{2} v_{2} w_{3} x_{1} w_{1}$.

(b) If, without loss of generality, $v_{1} \in R_{1}$ and we let $u_{1} \rightarrow$ $v_{1}$, then $x_{1} \rightarrow u_{1}, C_{3} \Rightarrow u_{1}$, and $u_{2} \in R_{2}$.

(i) Let $x_{1} \rightarrow v_{1}$.
Assume that $\left\{x_{2}, x_{3}\right\} \subset R_{2}$. Then $Y=\left\{w_{1}, w_{2}\right\}$ and $Z=$ $\left\{v_{2}\right\}$. In addition, $i_{l}(D) \leq 1$ implies $\left(V\left(C_{3}\right) \cup Z\right) \Rightarrow R_{1}$.

If $x_{2} \rightarrow u_{2}$ or $x_{3} \rightarrow u_{2}$, say, $x_{2} \rightarrow u_{2}$, then $u_{2} \Rightarrow$ $\left(\left\{x_{3}, v_{2}\right\} \cup V\left(C_{3}\right)\right)$ and $x_{3} \rightarrow\left(\left\{v_{2}\right\} \cup V\left(C_{3}\right)\right)$. Since $i_{l}(D) \leq 1$, $x_{2} \rightarrow u_{3}$ or $x_{2} \rightarrow v_{3}$. In the former case there exist two complementary cycles $x_{3} v_{2} u_{1} x_{3}$ and $w_{1} x_{2} u_{3} x_{1} w_{2} u_{2} v_{3} w_{3} v_{1} w_{1}$, and in the latter case two complementary cycles are $x_{3} v_{2} u_{1} x_{3}$ and $w_{1} x_{2} v_{3} x_{1} w_{2} u_{2} w_{3} u_{3} v_{1} w_{1}$.

If $u_{2} \rightarrow\left\{x_{2}, x_{3}\right\}$, then $\left\{x_{2}, x_{3}\right\} \rightarrow\left(\left\{v_{2}\right\} \cup V\left(C_{3}\right)\right)$. In addition, if $u_{2} \rightarrow v_{2}$, we have the complementary cycles $u_{2} v_{2} x_{1} u_{2}$ and $w_{1} x_{2} u_{3} v_{1} w_{2} x_{3} v_{3} w_{3} u_{1} w_{1}$; if $v_{2} \rightarrow u_{2}$ and there exists an arc from $Y$ to $Z$, say, $w_{1} \rightarrow v_{2}$, then we have the complementary cycles $C_{1}=w_{1} v_{2} x_{1} w_{1}$ and $C_{2}=$ $x_{2} u_{3} v_{1} w_{2} u_{2} x_{3} v_{3} w_{3} u_{1} x_{2} ;$ if $v_{2} \rightarrow\left(\left\{u_{2}\right\} \cup Y\right)$, then $V\left(C_{3}\right) \Rightarrow v_{2}$, and we have the complementary cycles $C_{1}=v_{2} w_{1} x_{2} v_{2}$ and $C_{2}=w_{2} u_{2} x_{3} u_{3} v_{3} w_{3} x_{1} u_{1} v_{1} w_{2}$.

Now assume that $x_{2} \notin R_{2}$ or $x_{3} \notin R_{2}$. Then $Y=\left\{x_{2}, x_{3}\right\}$ and $v_{2} \in R_{2}$. Otherwise, there exists one vertex $x \in R_{2}$ such that $d_{D}^{-}(x) \geq 6$, a contradiction. Therefore, let, without loss of generality, $Z=\left\{w_{1}\right\}$. Then we have $R_{2}=\left\{u_{2}, v_{2}, w_{2}\right\},(Z \cup$ $\left.V\left(C_{3}\right)\right) \Rightarrow\left\{x_{1}, u_{1}\right\}$, and $w_{2} \rightarrow\left\{u_{2}, v_{2}\right\}$. Let, without loss of generality, $u_{2} \rightarrow v_{2}$. This leads to the contradiction $d_{D}^{-}\left(v_{2}\right) \geq$ 6.

(ii) Let $v_{1} \rightarrow x_{1}$. Then $v_{2} \in R_{2}$ and $\left(Z \cup V\left(C_{3}\right)\right) \Rightarrow v_{1}$. Let, without loss of generality, $u_{2} \rightarrow v_{2}$. If $Y=\left\{w_{1}, w_{2}\right\}$, let, without loss of generality, $Z=\left\{x_{2}\right\}, x_{3} \in R_{2}$; then $v_{2} \rightarrow x_{3} \rightarrow u_{2}, R_{2} \Rightarrow\left(V\left(C_{3}\right) \cup Z\right)$, and $\left(Z \cup V\left(C_{3}\right)\right) \Rightarrow R_{1}$. It is evident that $u_{2} x_{2} v_{1} u_{2}$ and $w_{1} x_{3} w_{3} x_{1} w_{2} v_{2} u_{3} v_{3} u_{1} w_{1}$ are complementary cycles in $D$. If $Y=\left\{x_{2}, x_{3}\right\}$, let, without loss of generality, $Z=\left\{w_{1}\right\}$; then $v_{2} \rightarrow w_{2} \rightarrow u_{2}$. This leads to the contradiction $d_{D}^{+}\left(w_{2}\right) \geq 6$.

(2) Assume that $\left|R_{1} \cap V_{4}\right|=2$. Let, without loss of generality, $R_{1}=\left\{x_{1}, x_{2}, u_{1}\right\}$ and $Y=\left\{v_{1}, v_{2}\right\}$.

(a) If $Z=\left\{x_{3}\right\}$, then $R_{2}=\left\{u_{2}, w_{1}, w_{2}\right\}$ and $\left\{w_{1}, w_{2}\right\} \rightarrow u_{2}$. This leads to the contradiction $d_{D}^{-}\left(u_{2}\right) \geq 6$.

(b) If $Z=\left\{u_{2}\right\}$, then $R_{2}=\left\{x_{3}, w_{1}, w_{2}\right\},\left\{w_{1}, w_{2}\right\} \rightarrow x_{3}$, $R_{2} \Rightarrow\left(V\left(C_{3}\right) \cup Z\right)$, and $\left(V\left(C_{3}\right) \cup Z\right) \Rightarrow R_{1}$. This leads to the complementary cycles $C_{1}=w_{1} u_{2} x_{1} w_{1}$ and $C_{2}=$ $v_{1} w_{2} u_{3} x_{2} v_{2} x_{3} v_{3} w_{3} u_{1} v_{1}$.

(c) If, without loss of generality, $Z=\left\{w_{1}\right\}$, then $R_{2}=$ $\left\{x_{3}, u_{2}, w_{2}\right\}, w_{2} \rightarrow\left\{u_{2}, x_{3}\right\}$, and $u_{2} \rightarrow x_{3}$. This leads to $R_{2} \Rightarrow\left(V\left(C_{3}\right) \cup Z\right)$ and $\left(V\left(C_{3}\right) \cup Z\right) \Rightarrow R_{1}$.

If $u_{1} \rightarrow x_{1}$ or $u_{1} \rightarrow x_{2}$, say, $u_{1} \rightarrow x_{1}$, then $x_{2} \Rightarrow u_{1}$ and $\left(Z \cup V\left(C_{3}\right)\right) \Rightarrow\left\{u_{1}, x_{2}\right\}$. This leads to the complementary cycles $C_{1}=u_{2} w_{1} x_{1} u_{2}$ and $C_{2}=v_{1} w_{2} u_{3} x_{2} v_{2} x_{3} v_{3} w_{3} u_{1} v_{1}$.

If $\left\{x_{1}, x_{2}\right\} \rightarrow u_{1}$, then $Z \cup V\left(C_{3}\right) \Rightarrow\left\{x_{1}, x_{2}\right\}$. This leads to the complementary cycles $C_{1}=x_{3} w_{1} u_{1} x_{3}$ and $C_{2}=$ $v_{1} u_{2} v_{3} w_{3} x_{1} v_{2} w_{2} u_{3} x_{2} v_{1}$.

Thus we finish proof of case $(|Z|,|Y|)=(1,2)$.

Case 3. One has $|Z|=1$ and $|Y|=3$. Then $Y=V_{4}=$ $\left\{x_{1}, x_{2}, x_{3}\right\}$.

The case is equivalent to the case of " $|Y|=2, x_{3} \in R_{2}$, and $x_{3} \rightarrow R_{2} \backslash\left\{x_{3}\right\}$," which has been proved in Case 2 .

\section{Concluding Remarks}

In this paper, we proved the existence of complementary cycles in a kind of irregular multipartite tournaments. 
Problems on complementary cycles in irregular multidigraphs have important significance.

In 2002, Volkmann gave a new type of connectivity condition for a multipartite tournament to be cycle complementary. They proposed the following conjecture.

Conjecture 13 (see Volkmann [16] 2002). Let D be a multipartite tournament. If $k(D) \geq \alpha(D)+1$, then $D$ is cycle complementary, unless $D$ is a member of a finite family of multipartite tournaments.

In 2010, Li et al. [17] proved every $(\alpha(D)+1)$-strong $n$ partite tournament $D$ with $n \geq 6$ contains two disjoint cycles which contain vertices from exactly 3 and $n-3$ partite sets, respectively, unless it is isomorphic to $T_{7}^{1}$.

The vertex set of a digraph $D$ is denoted by $V(D)$. A $c$ partite tournament is an orientation of a complete $c$-partite graph. A multipartite tournament $D$ is called weakly cycle complementary if there exist two vertex disjoint cycles $C_{1}$ and $C_{2}$ such that $V\left(C_{1}\right) \cup V\left(C_{2}\right)$ contains vertices of all partite sets of D. In 2008, Volkmann and Winzen [18] proved all 3connected c-partite tournaments with $c \geq 3$ that are weakly cycle complementary. In 2011, He and Wang [19] proved all 3-partite tournaments that are weakly cycle complementary.

In 2008, Volkmann and Winzen [18] also proposed the following problem.

Problem 14 (see Volkmann and Winzen [18] 2008). Characterize all 2-strong $c$-partite tournaments $D$ with $c \geq 3$ and $|V(D)| \geq 6$ that are weakly cycle complementary.

All these problems may stimulate our further study for complementary cycles of digraphs.

\section{Competing Interests}

The authors declare that there is no conflict of interests regarding the publication of this paper.

\section{Acknowledgments}

This work is supported by the Natural Science Funds of China (no. 11501490 and no. 61572292) and by the Natural Science Foundation of Shandong Province (no. ZR2015AM006).

\section{References}

[1] K. B. Reid, "Two complementary circuits in two-connected tournaments," Annals of Discrete Mathematics, vol. 27, pp. 321334, 1985.

[2] Z. M. Song, "Complementary cycles of all lengths in tournaments," Journal of Combinatorial Theory B, vol. 57, no. 1, pp. 1825, 1993.

[3] A. Yeo, "Diregular c-partite tournaments are vertex-pancyclic when $c \geq 5$," Journal of Graph Theory, vol. 32, no. 2, pp. 137-152, 1999.

[4] L. Volkmann, "Complementary cycles in regular multipartite tournaments," The Australasian Journal of Combinatorics, vol. 31, pp. 119-134, 2005.
[5] L. Volkmann, "Complementary cycles in regular multipartite tournaments, where one cycle has length four," Kyungpook Mathematical Journal, vol. 44, no. 2, pp. 219-247, 2004.

[6] Z. He, T. Korneffel, D. Meierling, L. Volkmann, and S. Winzen, "Complementary cycles in regular multipartite tournaments, where one cycle has length five," Discrete Mathematics, vol. 309, no. 10, pp. 3131-3149, 2009.

[7] L. Volkmann, "Complementary cycles in almost regular multipartite tournaments, where one cycle has length four," Discrete Applied Mathematics, vol. 161, no. 13-14, pp. 2169-2177, 2013.

[8] Y. Bai, H. Li, and W. He, "Complementary cycles in regular bipartite tournaments," Discrete Mathematics, vol. 333, pp. 1427, 2014.

[9] Z. He, L. Volkmann, and Y. Wang, "Complementary cycles in almost regular multipartite tournaments," Ars Combinatoria, vol. 113A, pp. 201-224, 2014.

[10] A. Yeo, Semicomplete multipartite digraphs [Ph.D. thesis], Odense University, 1998.

[11] J. A. Bondy, "Diconnected orientation and a conjecture of Las Vergnas," Journal London Mathematical Society, vol. 14, pp. 277282, 1976.

[12] A. Yeo, "How close to regular must a semicomplete multipartite digraph be to secure Hamiltonicity?" Graphs and Combinatorics, vol. 15, no. 4, pp. 481-493, 1999.

[13] M. Tewes, "Almost all regular c-partite tournaments with $c \geq 5$ are vertex pancyclic," Discrete Mathematics, vol. 242, no. 1-3, pp. 201-228, 2002.

[14] G. Gutin and A. Yeo, "Note on the path covering number of a semicomplete multipartite digraph," Journal of Combinatorial Mathematics and Combinatorial Computing, vol. 32, pp. 231-237, 2000.

[15] L. Volkmann and S. Winzen, "Almost regular multipartite tournaments containing a Hamiltonian path through a given arc," Discrete Mathematics, vol. 285, no. 1-3, pp. 267-278, 2004.

[16] L. Volkmann, "Cycles in multipartite tournaments: results and problems," Discrete Mathematics, vol. 245, no. 1-3, pp. 19-53, 2002.

[17] S. Li, W. Meng, and Y. Guo, "Three supplements to Reid's theorem in multipartite tournaments," Discrete Applied Mathematics, vol. 158, no. 4, pp. 340-348, 2010.

[18] L. Volkmann and S. Winzen, "Weakly complementary cycles in 3-connected multipartite tournaments," Kyungpook Mathematical Journal, vol. 48, no. 2, pp. 287-302, 2008.

[19] Z. He and Y. Wang, "Weakly cycle complementary 3-partite tournaments," Graphs and Combinatorics, vol. 27, no. 5, pp. 669$683,2011$. 


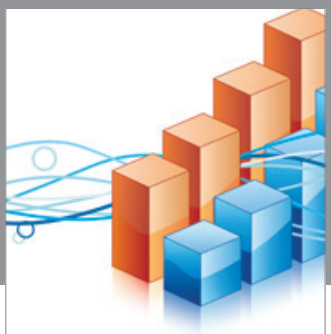

Advances in

Operations Research

vatem alat4

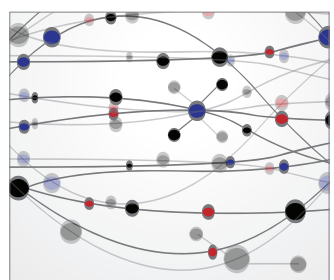

\section{The Scientific} World Journal
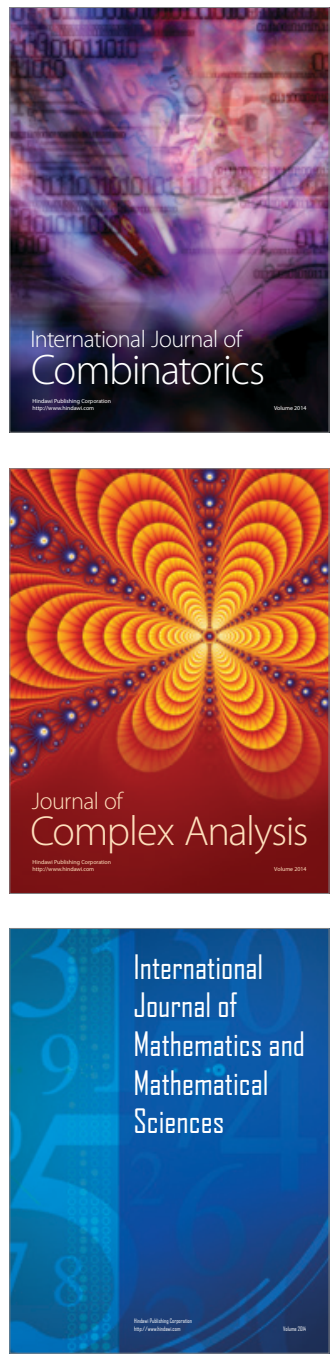
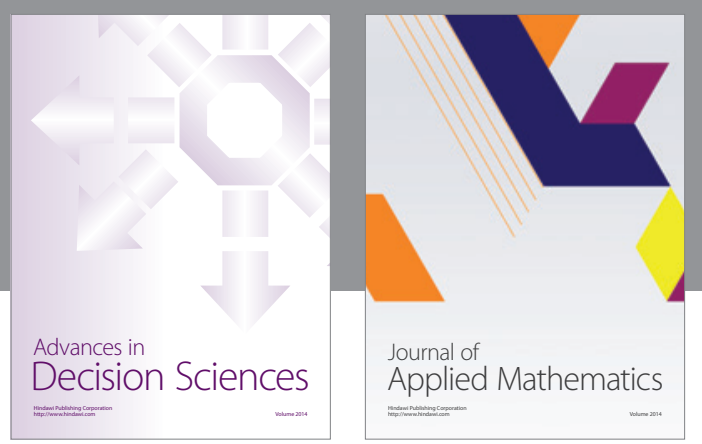

Algebra

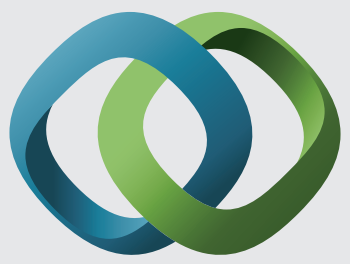

\section{Hindawi}

Submit your manuscripts at

http://www.hindawi.com
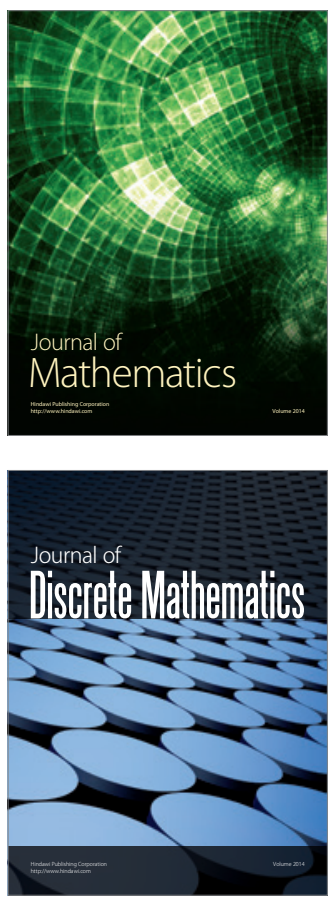

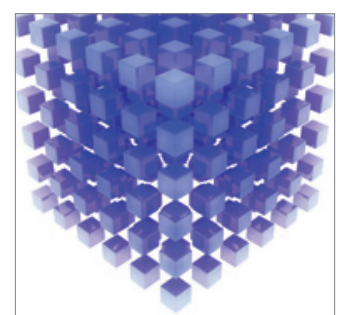

Mathematical Problems in Engineering
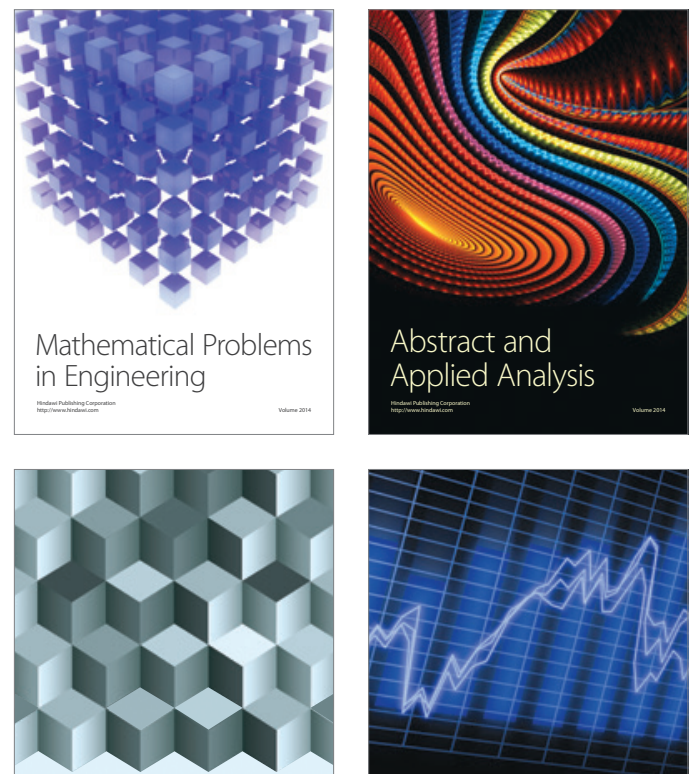

Journal of

Function Spaces

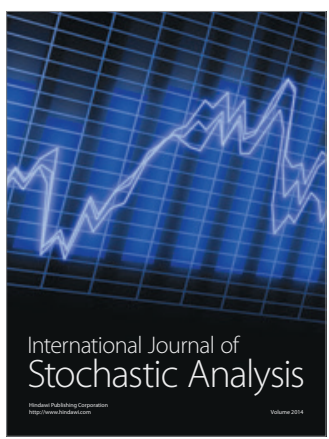

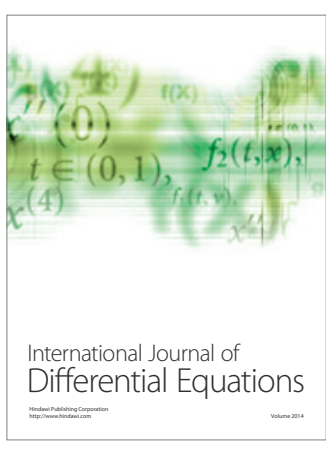
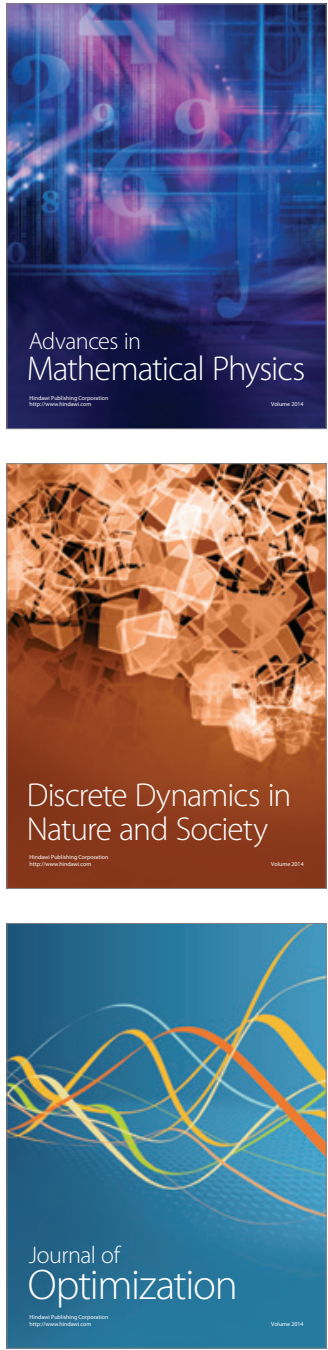